\title{
The Heart-sign of Bilateral Medial Medullary Infarction
}

Pedro Radalle Biasi ${ }^{1 *}$, Grégori Manfroi ${ }^{2}$, Timóteo Abrantes de Lacerda Almeida ${ }^{1}$, Luciano Bambini Manzato ${ }^{1,3}$

${ }^{1}$ Department of Neurosurgery, São Vicente de Paulo Hospital, Passo Fundo, Brazil

${ }^{2}$ University of Passo Fundo - School of Medicine, Passo Fundo, Brazil

${ }^{3}$ Service of Neurology and Neurosurgery, Passo Fundo, Brazil

*Corresponding author: Pedro Radalle Biasi, Department of Neurosurgery, São Vicente de Paulo Hospital, Passo Fundo, Brazil, Tel: +555496172514; E-mail: pedrobiasi@doctor.com

Rec date: Apr 19, 2016; Acc date: Jun 21, 2016; Pub date: Jun 24, 2016

Copyright: (C) 2016 Biasi PR, et al. This is an open-access article distributed under the terms of the Creative Commons Attribution License, which permits unrestricted use, distribution, and reproduction in any medium, provided the original author and source are credited.

\begin{abstract}
The Bilateral Medial Medullary Infarction presents with progressive tetraparesis, bilateral profound sensory loss, dysphagia and dysarthria, progressing to respiratory failure, which can be misdiagnosed with Guillain-Barré syndrome. The main cause its vertebral artery atherosclerosis and thrombosis affecting the anteromedial (supplied by branches of vertebral artery and anterior spinal artery) and anterolateral (fed by short and long transverse branches of vertebral artery) territories of medulla bilaterally. The diagnosis is facilitated by Diffusion Weighted Image and ADC-map on MRI, which shows a V-shape infarction, or, as known, the "heart sign" (Figure 1 and Figure 2 ), which is typical of this disease. The outcome is poor, with high mortality.
\end{abstract}

Keywords: Medullary Infarction; Stroke; Guillain-Barré syndrome; Magnetic resonance imaging

\section{Introduction}

Bilateral Medial Medullary Infarction (BMMI) is a rare entity. Even during the magnetic resonance imaging (MRI) era early diagnostic remains a challenge, because the clinical presentation can mimic others pathologies such as Guillain-Barrés syndrome (GBS). Therefore a high suspicion is needed to close such diagnose. We present a case of a male patient with bilateral medial medullary infarction that was initially suspected of GBS.

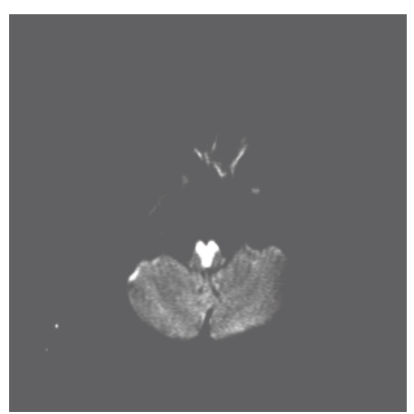

Figure 1: Axial DWI-MR Image shows the "Heart-sign" - the hyperintense signal at the anteromedial and anterolateral territories of medulla oblongata, compatible with acute stroke.

\section{Case Report}

A male patient, 82-year-old, presenting with acute paraparesis rapidly evolved to tetraparesis, respiratory failure and coma. His deep tendon reflexes were initially normal, also with flexor plantar reflex.
Cranial nerve examination revealed absence of oculocephalic reflex. Past medical history includes hypertension.

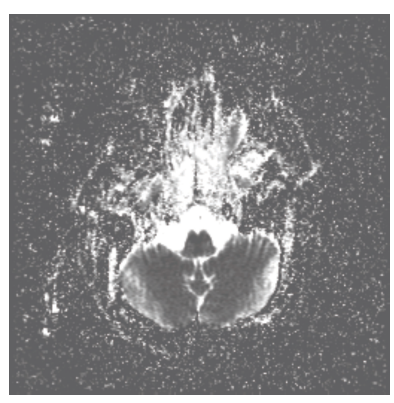

Figure 2: Axial ADC-MR Image shows the hypo-intense signal and confirms acute stroke at medulla oblongata.

Brain CT was normal, being preceded to therapeutic plasmapheresis, without neurological improvement. After few days he presented with enhanced deep tendon reflexes and Babinski signal becomes present. Then, we proceed to a brain MRI, which shows medullary infarction on both sides of midline, heart-shaped, configuring Bilateral Medial Medullary Infarction (Figure 1 and Figure 2). Cerebral MR angiography reveals no abnormality on posterior circulation arteries. Patient died in consequence of pneumonia.

\section{Discussion}

Bilateral Medial Medullary Infarction is a rare and severe stroke, accounting for less than $1 \%$ of vertebrobasilar strokes [1,2]. Diagnoses can be delayed due to the heterogeneity of clinical presentation that includes tetraplegia, dysphagia, sensory loss, tongue weakness and stridor [3, 4]. A higher suspicion is needed, because the condition can be misdiagnosed as GBS, the most common cause of ascending acute flaccid paralysis [5], especially in a case as the described by us. 
Page 2 of 2

Occlusion of the Vertebrobasilar system or anterior spinal artery or also their intrinsic penetrating branches seems to be the underlying vascular pathology $[3,5]$. Prior to the era of MRI, bilateral medial medullary infarction (BMMI) was an obscure pathology $[5,6]$. The characteristic brain MRI finding of "heart appearance" at Diffusion Weighted Imaging (DWI) has been described and is believed to appear when lateral and posterior territory of the medulla oblongata's blood supply are involved $[2,4,7]$. DWI-MRI is the current gold standard in the diagnosis of acute ischemic stroke and can detect ischemic changes within minutes of the onset of symptoms, showing up hyper-intense signal areas on DWI with corresponding hypo-intense areas on Diffusion Co-efficient (ADC) map1.

The outcomes are poor, with a severe mortality and morbidity, in a systematic review including 38 patients with BMMI proven by MRI, one fifth of patients died and two third of survivors remained dependent [3]. Therefore, differential diagnosis with GBS is essential, considering the management that should be given for these different pathologies [8]. A key-point to differentiate both syndromes is the evolution of the clinical presentation, whereas GBS have and sub-acute evolution while BMMI has a more acute presentation $[3,5,8]$.

\section{Conclusion}

Therefore when facing patients with rapid GBS-like syndrome, we must remember BMMI, a rare stroke subtype, with a typical MRI finding, but that requires a high degree of suspicion, both as neurologist and as radiologist. Because the approaches of those two pathologies are completely different, early diagnosis is critical.

\section{References}

1. Jawad RA, Rana AI, Sultan F, Saeed MA (2013) Bilateral medial medullary infarction: A case report. J Pak Med Assoc 63: 387-389.

2. Kumral E, Afsar N, Kirbas D, Balkir K, Ozdemirkiran T (2002) Spectrum of medial medullary infarction: clinical and magnetic resonance imaging findings. J Neurol 249: 85-93.

3. Pongmoragot J, Parthasarathy S, Selchen D, Saposnik G (2013) Bilateral medial medullary infarction: a systematic review. J Stroke Cerebrovasc Dis 22: 775-780.

4. Tai MLS, Katiman E, Rahmat K, Tan CT (2012) Acute bilateral medial medullary infarct with hypoplastic vertebral artery. Clin Neurol Neurosurg [Internet] 114:1365-1367.

5. Ma L, Deng Y, Wang J, Du F, Xia F, et al. (2011) Bilateral medial medullary infarction presenting as Guillain-Barré-like syndrome. Clin Neurol Neurosurg 113: 589-591.

6. Toyoda K, Hasegawa Y, Yonehara T, Oita J, Yamaguchi T (1992) Bilateral medial medullary infarction with oculomotor disorders. Stroke 23: 1657-1659.

7. Ishizawa K, Ninomiya M, Nakazato Y, Yamamoto T, Araki N (2012) "Heart appearance" infarction of the pons: A case report. Case Rep Radiol ID: 690903.

8. Torabi AM (2013) Bilateral medial medullary stroke: a challenge in early diagnosis. Case Rep Neurol Med ID: 274373. 